\title{
Disk-based program swapping in 8080-based microcomputers
}

\author{
PAUL G. NEUMANN \\ University of Miami, Miami, Florida 33124
}

\begin{abstract}
Microcomputers, like large-scale computer systems, can benefit from executive routines which can interrupt a running program, save the program and its environment on a disk, and later reload the program and resume execution at the point of interruption. The process is discussed conceptually, and the specific routines for accomplishing the tasks in a North Star DOS environment are presented and documented.
\end{abstract}

Large-scale computer systems gain a great deal in flexibility and efficiency through the use of executive functions that swap-out low-priority programs or programs that are waiting for $\mathrm{I} / \mathrm{O}$ in favor of highpriority programs or programs for which the $I / O$ has been completed. When the priority of the swapped-out program comes to the top of the stack again, or when the $\mathrm{I} / \mathrm{O}$ has been completed, the program is swapped in, and execution resumes where it was interrupted. The process of swapping out, and later, swapping in, involves saving the program and its environment on a storage medium that allows rapid transfer of information, but which is not part of the working memory available to the CPU. The fastest way to accomplish this is to store the program and environment in an external memory, so that swapping in and out can be accomplished at memory read/write speeds. Alternatively, it may be accomplished by utilizing high-speed disk units as the storage medium. Obviously, the executive routines must utilize an algorithm for determining whether the double swap is more efficient than dead time in a particular situation.

Although there are microcomputers for which timesharing systems have been written, they are based on residence of programs in assigned partitions. A task remains resident in an assigned partition until complete. Under most circumstances, this is a perfectly acceptable way of going about things. Since microcomputers have no peripheral processors, nothing is gained by swapping out a program that is waiting for $\mathrm{I} / \mathrm{O}$. In fact, a great deal is lost since the same CPU that must perform the $\mathrm{I} / \mathrm{O}$ will be required to perform the swap, which results in time lost.

There are, however, circumstances involving priority that do not follow the general rule that swapping is inefficient in microcomputers. Consider a system that is used at times for "batch" data processing, which at

Requests for reprints should be sent to the author at the Laboratory for On-Line Acquisition of Data, Department of Psychology, University of Miami, Coral Gables, Florida 33124. other times may be dedicated to real-time laboratory control and/or data acquisition. There are times when a long batch job might be partially completed before the system must be dedicated to the laboratory control task. In such cases, it is efficient and convenient to utilize the computer time for the batch job as long as possible, interrupt the execution and save the program and its environment, and when the computer again becomes available, reload it and resume execution. Our system software accomplishes just that. Programs may be interrupted and saved on a minidisk, and execution may resume at any time in the future. It is also an invaluable tool during programming, when the program development software often must be overlayed with another subsystem to permit testing the program under development. Using this technique, a single command saves the program development subsystem, and another single command restores it after the program has been tested, eliminating reloading software, program files, and so on.

Since the specific software is not compatible with many systems, the technique will first be discussed in conceptual terms. The software used in our laboratory hardware configuration will be discussed also. The latter should be easily adapted to any 8080-based microcomputer with a floppy-disk or minifloppy-disk system.

Basic requirements are that everything critical to the execution of the program must be saved, and nothing may be altered during the process of saving it. The first requirement may be met on a general basis by the simple technique of saving all memory locations that are normally accessible to the operating system and programs under its control, that is, by saving a complete memory image. The second requirement requires a bit more consideration. The CPU will be used for the swapping operation, so obviously, the swap must either save the contents of all registers or be performed at a point at which the registers contain no useful information. Similarly, the stack must not be disturbed or it will contain the wrong sequence of return addresses upon resuming execution. This can be accomplished by relocating the working stack before any calls are exe- 
cuted. The most formidable problem, however, is how to obtain a "frozen" copy of the same disk operating system (DOS) that will be used to save the progam and its environment. Here, we reserve a small section of random-access memory and normally deny the system to access this partition to prevent it from storing data that will be overlaid in the swapping process. A copy of the DOS is then internally swapped into this reserved partition before any disk operations take place. The properly located copy is then used to perform the swap to the disk, which includes the frozen copy of the DOS.

The size of this partition varies from DOS to DOS, and according to how well the DOS is documented. Technically, only those portions of the DOS that will be involved in the actual data transfer need be copied, with the restriction that the segments must have a record size that is a multiple of the disk sector size (transfer to and from the disk actually takes place in sectors, usually 128 or 256 bytes in size). Since documentation of commercially available microcomputer software usually approaches the threshold of criminal negligence, it is often very difficult to determine the routines to be used, and even more difficult to determine if the routines are using scratch-pad memory locations elsewhere. The least frustrating approach is to swap the entire DOS.

Having saved the registers, relocated the stack, and frozen a copy of the DOS, we can proceed to save the environment in a straightforward fashion by transferring the memory contents to the disk, "stepping over" the working DOS, and continuing until all memory has been saved.

Resuming execution is the reverse process. The DOS is used to reload all memory locations from the disk except those utilized by the properly located and working copy of the DOS. The frozen copy of the DOS is swapped back to the original location, the registers and stack pointer are restored as necessary, and a return sequence is initiated by which control passes back to the routine that detected the command to interrupt execution. This routine, in turn, clears all flags and returns to its calling routine, denying that it has detected anything, and the program continues execution, perhaps a week or more later.

Translating the conceptual structure into working code is almost as simple as describing the process. First, it must be decided how the command will be implemented and where it will be detected. The operating system will have a routine that is called, usually between each $1 / 0$ operation, and of ten during compute-bound operations, the function of which is to detect an escape character that suspends execution and returns the system to a command mode. This routine is an excellent place to detect a single-special-character command to interrupt execution and save the environment.

In the North Star DOS, this routine detects a CONTROL-C. Its location will vary since it is an $\mathrm{I} / \mathrm{O}$ routine either written by the user or supplied by an intermediate vendor for a particular hardware configuration. It can easily be found, however, by examining locations $2017 \mathrm{H}$ and $2018 \mathrm{H}$. These locations contain the address of the routine in reverse-byte sequence (e.g., 2529 means location $2925 \mathrm{H}$ ). Once the routine is found, it can be disassembled, either with a disassembler or by hand. If it is the last of the $I / O$ routines located in the $1 / 0$ partition that extends from $2900 \mathrm{H}$ to $29 \mathrm{FFH}$, it can simply be extended into unused space. If not, it may be easiest to leave it where it is, place a new routine in lower memory, and change the jump address above to the location of the new routine. Either technique should also work with the CPM DOS, since the routine is located in the BIOS partition.

The command is implemented by deciding first upon the special characters that will command the interrupt and the reload processes. I used a simple stimulusselection technique and chose CONTROL-I $(09 \mathrm{H}$ on the keyboard) for the interrupt command and CONTROL-R $(12 \mathrm{H})$ to resume execution. The code $09 \mathrm{H}$ corresponds to the ASCII code for horizontal tab, and users with software that utilizes this tab function may wish to choose different codes. Whichever codes are chosen, the routine is expanded to look for those codes, as well as the original CONTROL-C, and to call the appropriate routine when detected. It terminates with a normal RET instruction. Be sure that this routine "CALL's" the routine that saves the environment. This operation places a return address in the stack, which is saved. Thus a return from the reloading routine transfers control back to the original routine.

Program 1 contains the instruction sequence for the swapping operations. On entry, the HL register pair is loaded with the origin of the North Star DOS, and the DE register pair is loaded with the origin of the reserved partition in high memory. The first address is a constant for standard North Star systems. The second varies according to the random-access memory available. In most systems, it is probably best to use low memory below the DOS. This allows the system to store source code and data up to the end of existing memory. In our system, however, this area is filled with system routines, and the highest 10 sectors are reserved. Having loaded these addresses, the first routine transfers the DOS to a safe partition, terminating when the incremented $\mathrm{H}$ register reaches $2 \mathrm{AH}$, indicating that it points 1 byte past the end of the DOS. Notice that no registers were saved. This is permissible in the North Star DOS since it clears all registers except the program counter and stack pointer before calling the CONTROL-C routine. In other systems, all registers should probably be "PUSH'ed" to the stack.

The next operation is to preserve the contents of the stack pointer, which is stored in the last 2 bytes of the domain of the frozen DOS. It cannot be "PUSH'ed" to the stack, because the user then has to know the location of the stack to find the location of the stack (somewhat like needing one's glasses to find one's glasses). 
Next, the registers are loaded with arguments to the DOS routines that transfer memory to the disk. This routine addresses the output to Disk Unit 2. This allows the program to access Unit 1 while a disk is inserted in Unit 2 in preparation for the interrupt. In single-drive systems, it is necessary to insert an I/O loop that waits for a character to be typed on the keyboard before proceeding. This gives the user time to insert a fresh disk in the unit before the interrupt routine begins sequentially accessing the disk with destructive write operations. It is also possible to insert a sequence that queries the disk directory for free space, creates a file of appropriate length in available space, and stores the environment in that file. This entails a lot of overhead, so we use an empty disk and write on the first $\mathrm{n}$ sectors. The stack must be relocated before calling the input routine.

The partition-saving routines now follow in sequence. The last routine is specific to the SOL-20 main frame and other main frames that use the standard Processor Technology VDM-1 video display board. When the last save operation is finished, the routine jumps to the system monitor reentry location, from which rebooting operations may be initiated.

A caution: during the saving operations, do not attempt to save the contents of the read-only memory that resides from E800H to EBFFH. This read-only memory is on-board the disk controller board, and certain operations of the disk controller are initiated simply by placing addresses within its domain on the bus address lines. Attempting to transfer the data in these locations results in issuing the disk controller erroneous commands to power up the drive motor, step the trackseek mechanism, lower the head, and so on. Curious computer-jocks who attempt to dump the contents of the read-only memory to unravel the mysteries of the DOS will discover that it creates inexplicable system crashes and initiates erratic behavior in the disk drives.
Turning off power to the drives prevents this and permits copying the read-only memory. During disk operations, however, stay out of its domain.

Having stored the program and its environment, we now turn to the problem of restarting the program. Program 2 illustrates the steps. First, the DOS is booted into memory, and the resume command is issued, whereupon control is transferred to Program 2. Program 2 initiates the read sequences, using the current copy of DOS to load memory. Some users may want to combine the save/load operations into a single set of routines entered with the read or write argument in register $B$. All other operations are identical and can be performed in the same sequence. After loading memory, the frozen copy of the DOS is transferred to the normal origin. At this point, users with single-drive systems will once again want to insert an input loop to permit placing the correct disk in the disk drive before resuming execution of the program. Be sure that the loop is placed before the stack pointer is restored. The stack pointer is restored, and the accumulator is zeroed out so that it does not accidentally contain a legal character. At this point, if the user "PUSH'ed" the contents of the other register pairs, they will be "POP'ed" in the reverse sequence. A retum is then initiated, which transfers control back to the command-detecting routine; it should then return to the calling routine.

These routines should also be easily translated into the appropriate instructions for the Z-80 CPU. Using the LDIR instruction simplifies the memory transfer of DOS.

The routines may be located anywhere in memory. Since all parameters remain in the registers, overlaying the code during the reloading operations has no effect in its execution, since the current instruction resides in the instruction register when the program counter corresponds to the address at which the current byte is being stored.

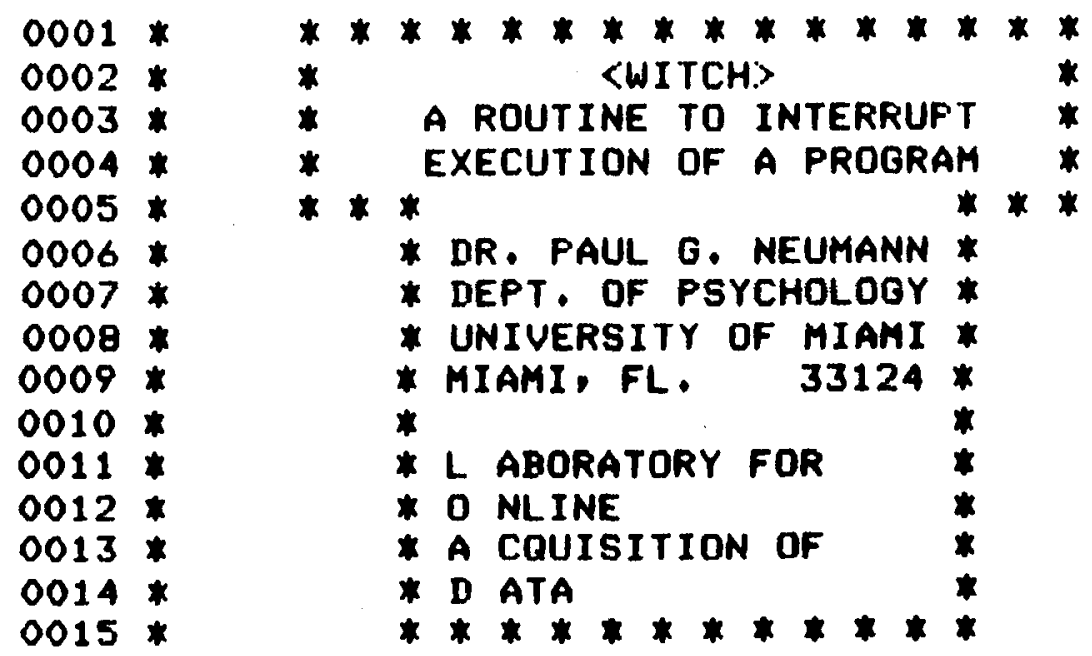




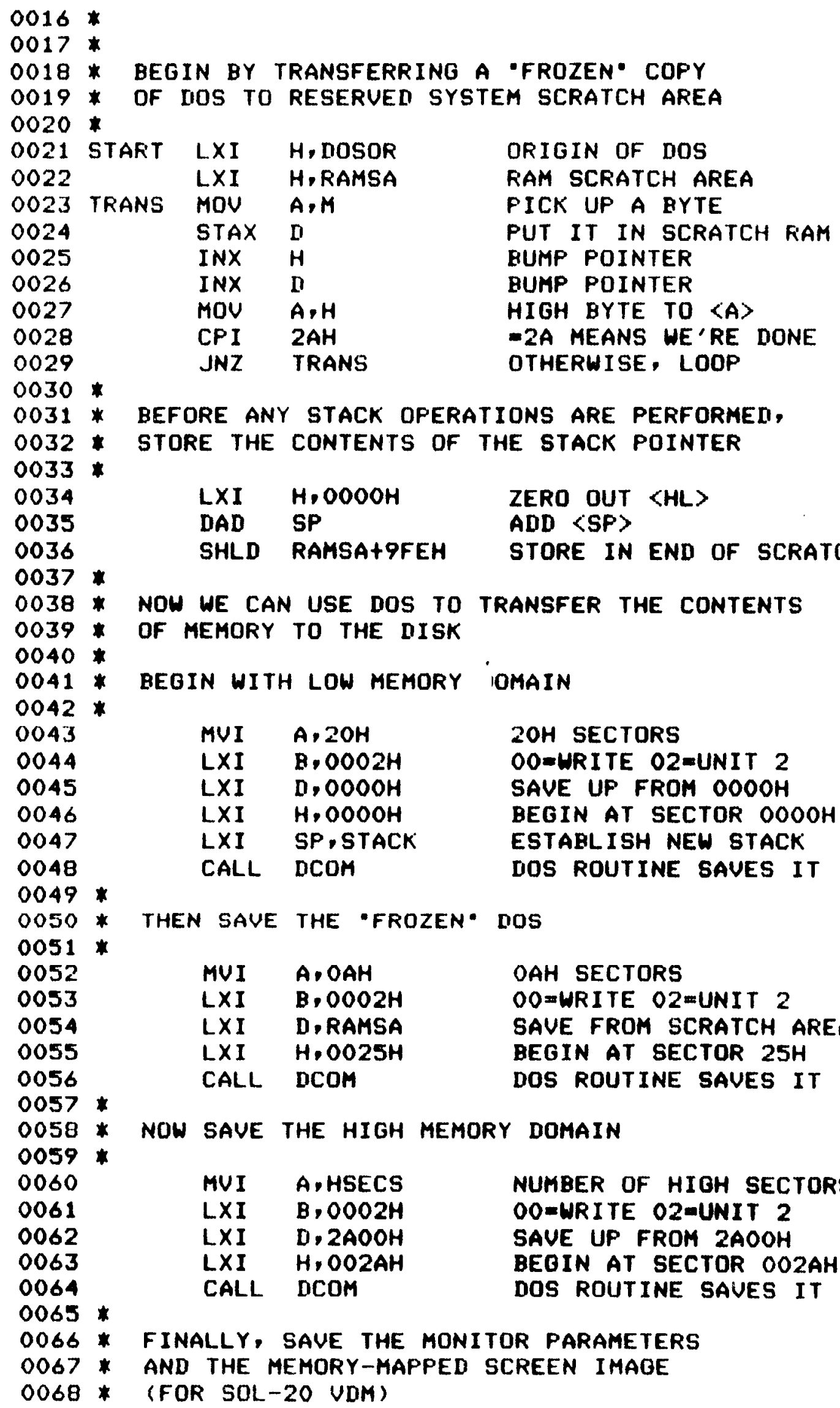

LXI H,O0OOH

DAD SP

SHLD RAMSAT9FEH

NOW WE CAN USE DOS TO TRANSFER THE CONTENTS

OF MEMORY TO THE DISK

BEGIN WITH LOW MEMORY IOMAIN

MUI $A, 20 \mathrm{H}$

LXI $\quad B .0002 \mathrm{H}$

LXI $\quad 0,0000 \mathrm{H}$

LXI H,OOOOH

LXI SP,STACK

CALL DCOM

then save the " frozen" dos

MUI A.OAH

LXI B, 0002H

LXI D.RAMSA

LXI H.0025H

CALL DCOM

NOW SAVE THE HIGH MEMORY DOMAIN

MUI A.HSECS

LXI B, 0002H

LXI D, 2AOOH

LXI H,OO2AH

CALL DCOM

2OH SECTORS

OO=WRITE $02=U N I T 2$

SAVE UP FROM $0000 \mathrm{H}$

BEGIN AT SECTOR OOOOH

ESTABLISH NEW STACK

DOS ROUTINE SAVES IT 


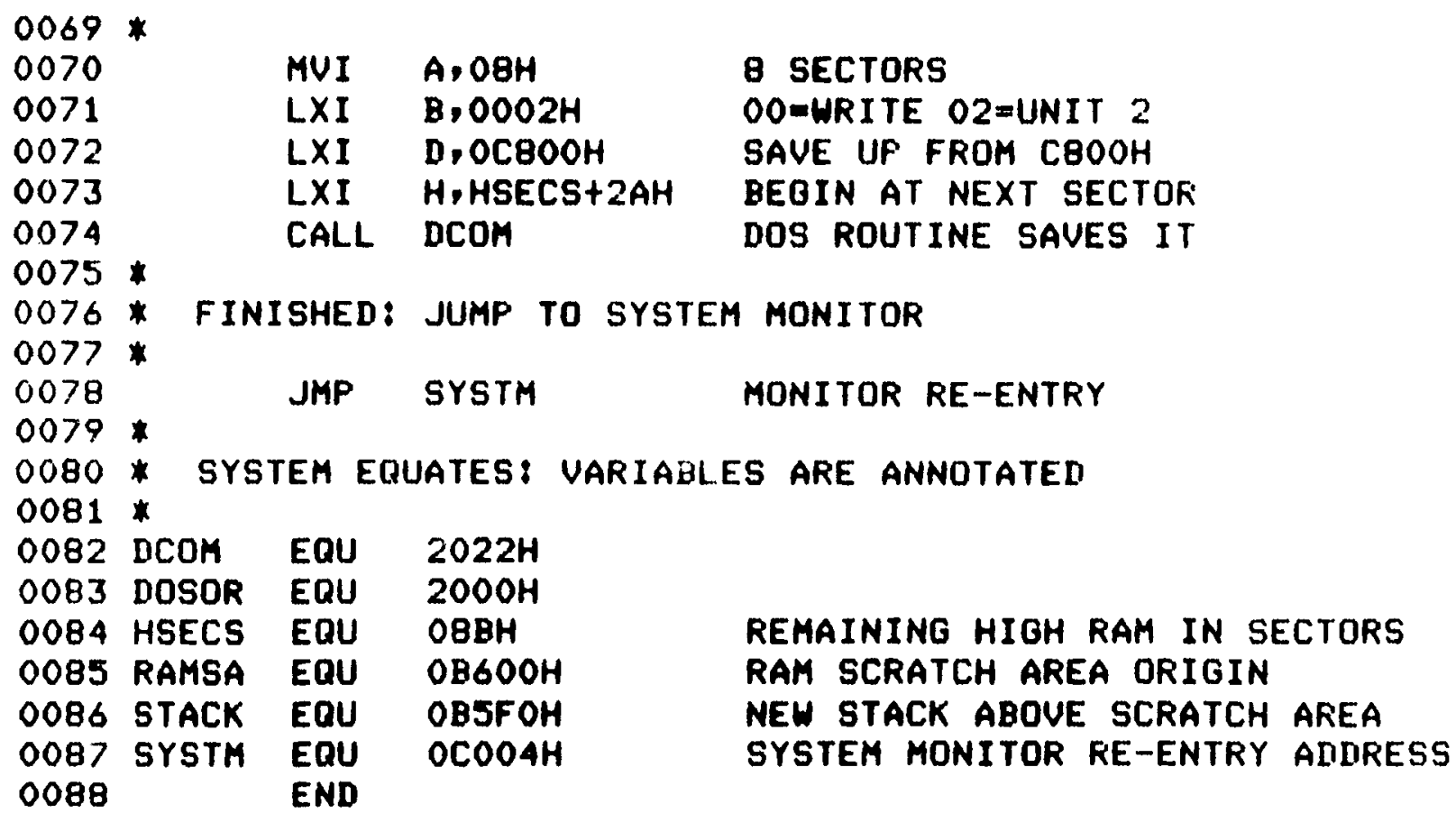

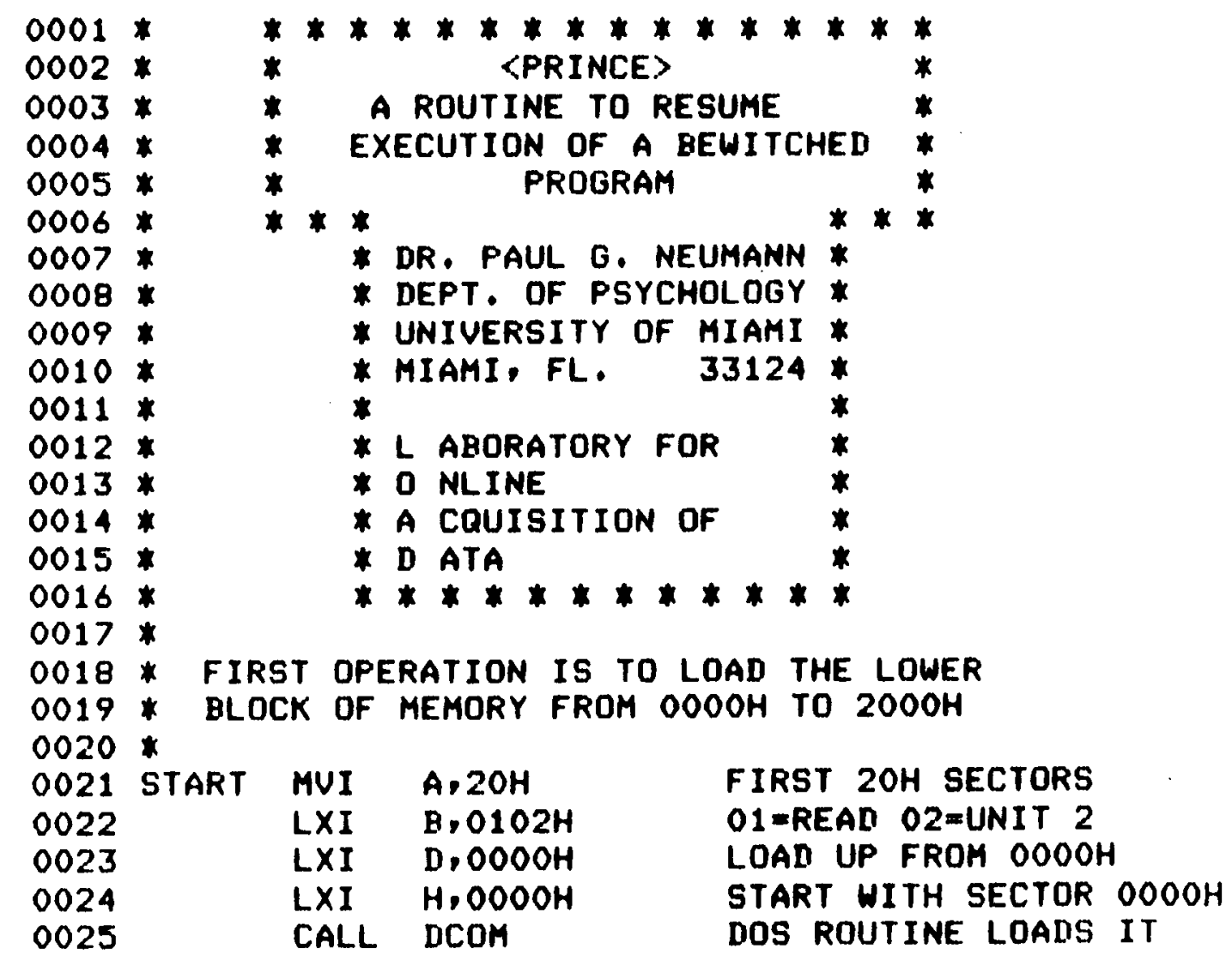




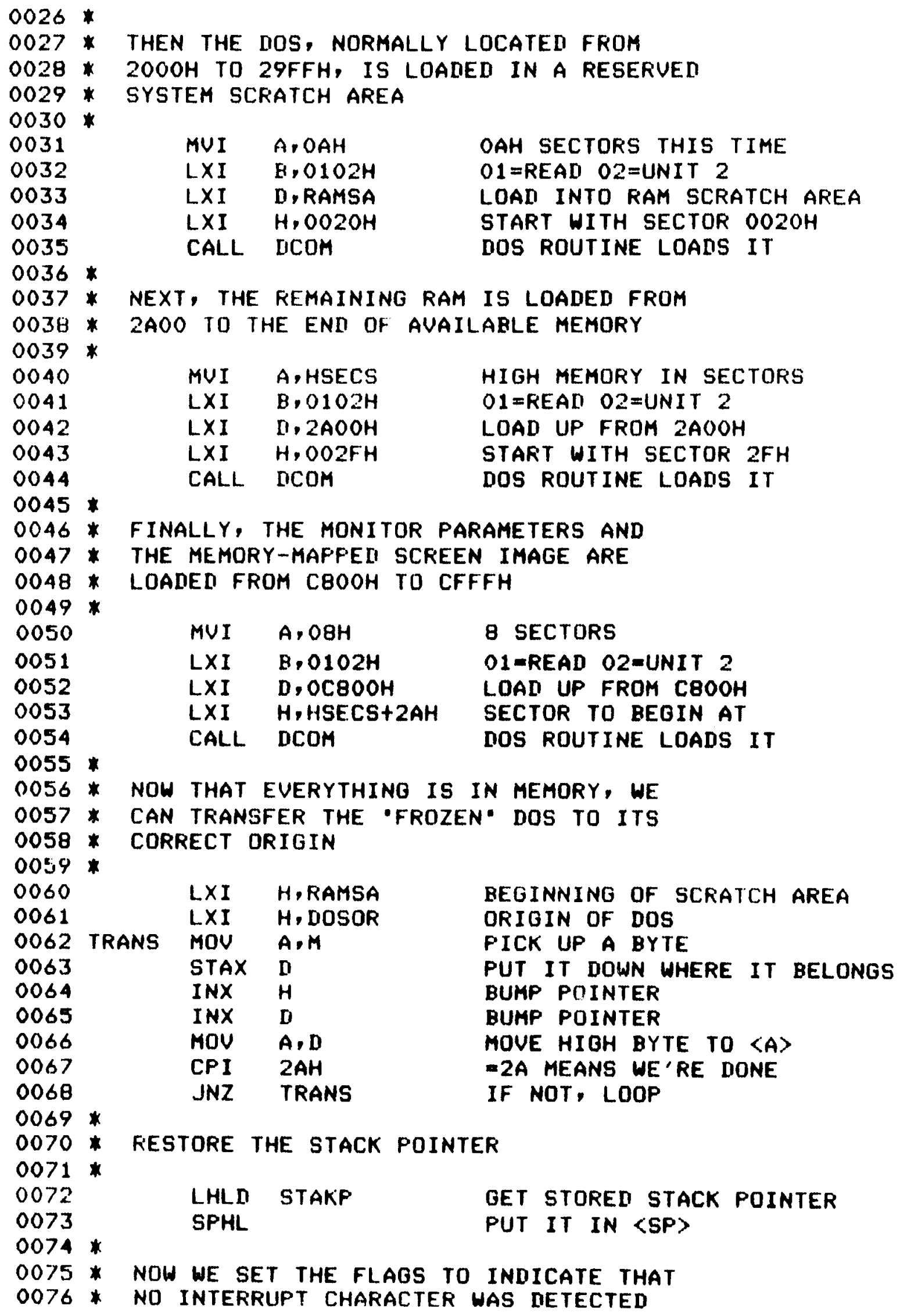


518 NEUMANN

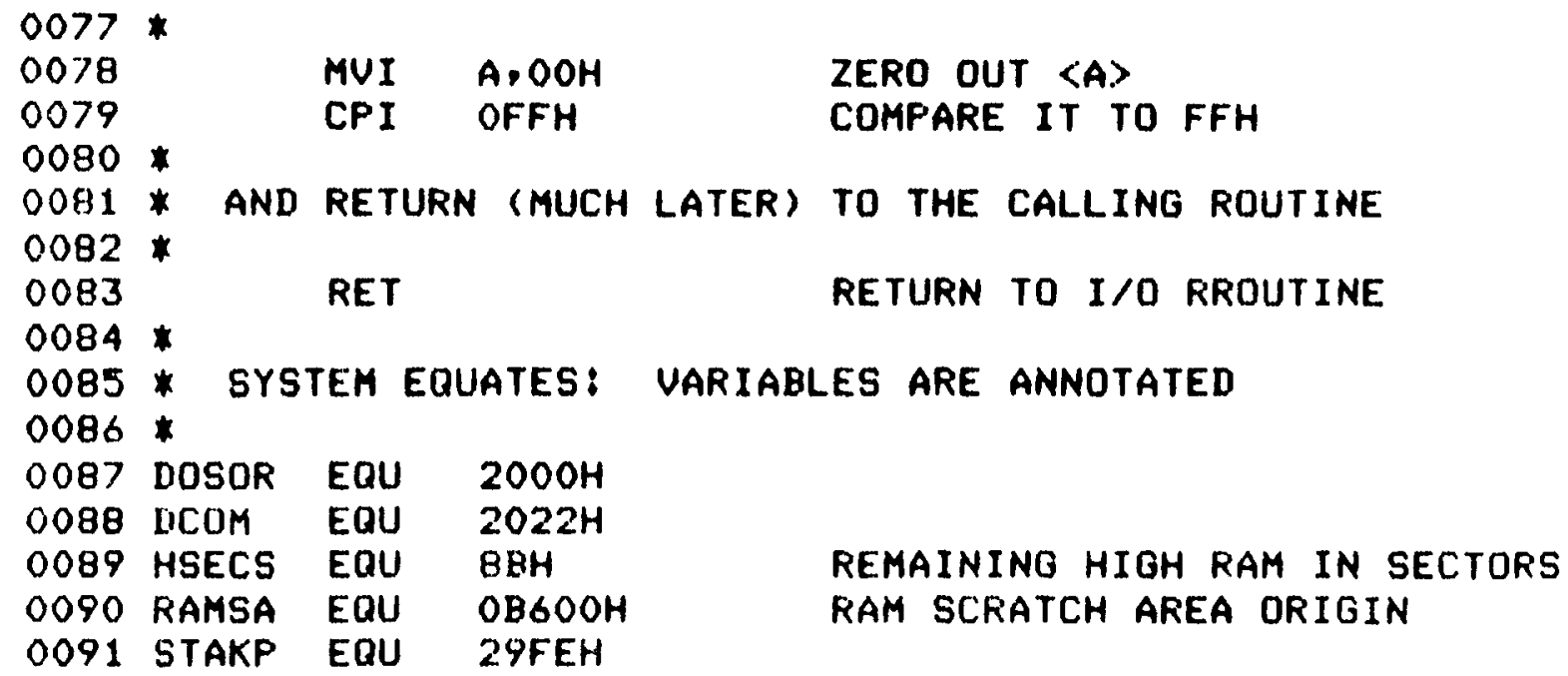

REMAINING HIGH RAM IN SECTORS RAM SCRATCH AREA ORIGIN 